\title{
Nach dem Deutschstudium in Afrika wird man - was? Eine Uberprüfung der Zielsetzungen der Deutschabteilung der Universität Lomé anhand einer Verbleibstudie
}

\author{
Eva Hamann \\ Universität Leipzig, Herder Institut, Beethovenstr. 15, 04107 Leipzig, Germany \\ E-mail: eva.hamann@uni-leipzig.de
}

\begin{abstract}
This paper reports on an investigation of the value of German Studies in eventual employment of students in this field. The objectives of German Studies in Africa have to include an indication of how inclusion in the curriculum is legitimized. Three approaches towards legitimization have developed since the establishment of German Departments at African Universities, namely (i) German Studies with a view to research and epistemological science, (ii) German in intercultural studies, and (iii) career-oriented German language learning. In this paper the implementation of these approaches will be examined on the basis of the objectives set out by the German Department at the University of Lomé. This will be done by discussing the results of a survey on the professional whereabouts of Togolese German Studies graduates to demonstrate the extent to which the objectives of the various approaches have been met. The paper concludes with a discussion of the implications of the findings for the programmes of the German Department at the University of Lomé.
\end{abstract}

Keywords: legitimization debate, distribution survey, teaching German in Africa, German language and literature studies in Africa

Schlüsselbegriffe: Legitimierungsdebatte, Verbleibstudie, Deutschunterricht in Afrika, Germanistik in Afrika

\section{Einleitung}

Der Beitrag elaboriert die Zielsetzungen beim Fremdsprachenunterricht mit Schwerpunkt auf Deutsch in einem afrikanischen Kontext. Dazu erfolgt zuerst ein kurzer Abriss über die Legitimierungsdebatte des Studienfachs Deutsch in Afrika, wobei drei Ansätze zur Legitimierung vorgestellt werden. Die Umsetzung der entwickelten theoretischen Ansätze wird anhand der Deutschabteilung an der Universität Lomé illustriert. Das Erreichen der Zielsetzungen der Abteilung wird anschließend mit Hilfe einer Verbleibstudie von togoischen Germanistikabsolventen überprüft. Zum Schluss erfolgt die Darstellung der Perspektiven für die Zielsetzungen an der Deutschabteilung. 


\section{Die Legitimierungsdebatte}

Die Debatte um die Legitimierung und Relevanz des Studienfachs Deutsch in Afrika, geführt von afrikanischen Auslandsgermanisten, spiegelt sich ab den 80er Jahren in afrikanischen Periodika wie Études Germano-Africaines und in deutschen Periodika wie Jahrbuch DaF wider. Dazu gehören u.a. Beiträge von Ihekweazu (1984, 1987), Sadji $(1983,1984)$ und Simo (1984, 1999). Dissertationen, wie die von Sow (1986) und Diop (2000) und Habilitationen, wie die von Ndong (1993) widmen sich ebenfalls dieser Debatte und der Situation des Deutschangebots in ihren jeweiligen Ländern. Die Frage nach der Notwendigkeit von Deutsch in Afrika und ob Germanistik einen gesellschaftlichen Stellenwert im Entwicklungsprozess der afrikanischen Länder beanspruchen kann, wird einstimmig mit ,ja“ beantwortet. Afrikanische Germanisten sehen die Teilbereiche der Auslandsgermanistik in ihrer Anpassung an örtliche Realitäten und Bedürfnisse etabliert und anerkannt. Nur über die Frage nach dem „Wie“ herrschen Meinungsunterschiede. Die drei wichtigsten Ansätze zur Relevanz der Germanistik in Afrika sind: erstens eine rein wissenschaftliche Germanistik mit emanzipatorisch-bewusstseinsbildendem Ziel, zweitens die interkulturelle Germanistik und drittens die berufsbezogene und fachsprachliche Fremdsprachenausbildung. Diese drei Ansätze werden im Folgenden näher erläutert.

Ein Pionier der forschungstheoretischen, rein wissenschaftlichen Germanistik in Afrika ist der deutsche Germanist Kreutzer (1989, 1998, 2009). Für ihn liegt die Legitimation der Germanistik in Afrika in der komparatistischen Literaturwissenschaft, die eine Rekonstruktion der sozialgesellschaftlichen Identität der einheimischen afrikanischen Länder anstrebt. Viele bei ihm in der École de Hanovre promovierte und habilitierte afrikanische Germanisten verfolgen seinen Ansatz weiter. So entwickelt z.B. Sow in seiner Dissertation das Konzept der Germanistik als Entwicklungswissenschaft, welches sich auf die Dependenztheorie stützt.

Der zweite viel diskutierte Ansatz der Auslandsgermanistik in Afrika ist das Konzept der interkulturellen Germanistik. Einer der Verfechter für die Germanistik als interkulturelle Wissenschaft, bzw. „kulturkontrastive Germanistik“, ist Wierlacher (1985). Auch sein Ansatz misst der Literaturwissenschaft eine entscheidende Rolle bei, berücksichtigt aber die Vermittlung der Kultur stärker. Afrikanische Germanisten wie Ihekweazu schlossen sich seinem Ansatz an. Andere, die dem ersten Ansatz anhängen, haben bei dem Konzept der interkulturellen Germanistik Bedenken, weil die kulturelle Partnerschaft von ungleichen Ausgangsbedingungen geprägt ist. Dadurch sei Akkulturation die Folge und die Rezeption fremdkulturellen Wissens von deutscher Seite nicht gegeben.

Ein Vorreiter für die Auslandsgermanistik als berufsbezogene und fachsprachliche Fremdsprachen-Ausbildung ist Sadji (1983). Er strebt die Förderung des Wissenschafts- und Technologietransfers durch die deutsche Sprache an und sieht in der Germanistik ein Mittel zur Gewinnung deutschen fach- und wissenschaftssprachlichen Wissens im afrikanischen Emanzipationsprozess - leider durch Initiative der Deutschen durch Entwicklungshilfe und nicht durch Afrikaner. Er schlägt eine Abteilung für berufsbezogene und fachsprachliche Ausbildung innerhalb der großen Germanistiken für andere Fakultäten in seinem Land vor.

Ein jüngerer Vertreter für diesen Ansatz ist Bedi (2006). Er schließt sich dem deutschen Germanisten Picht an, für den die berufsbezogene Germanistik eine politische Perspektive 
einnimmt, die viel mehr auf weltwirtschaftliche Verflechtungen als auf eine kulturelle Ebene fokussiert ist. Dieser dritte Ansatz des berufsbezogenen und fachsprachlichen Fremdsprachenangebots ist ausgerichtet auf erweiterte und praktische Tätigkeitsfelder. Diese Zielsetzung findet ihre Umsetzung z.B. im Studiengang LEA (Langues étrangères appliquées) in Frankreich, im ILEA (Institut des Langues Étrangères Appliquées) im Senegal, in dem Studiengang LATA (Langues Appliquées au Tourisme et aux Affaires) im Senegal und im GIL (Ghana Institut of Language) in Ghana. Bedi plädiert für eine stärkere Konzentration auf die Fachsprache Deutsch in Afrika, da die Fachkommunikation einen Bereich darstellt, für den der Bedarf im Zuge von Globalisierung, Mobilität und informationeller Vernetzung sprunghaft anwächst. Für Bedi entspricht die literaturwissenschaftliche Ausrichtung nicht mehr der Bedarfslage und den individuellen Bedürfnissen der Germanistikstudierenden.

\section{Zielsetzung der Deutschabteilung der Universität Lomé}

Wenden wir uns jetzt konkret einem Beispiel für die Überprüfung der Umsetzung der eben illustrierten Ansätze zu. Als Beispiel dient in diesem Beitrag die Deutschabteilung der Universität Lomé. Ein Blick in die Studienordnung für die Licence der Deutschabteilung weist ihre Zielsetzungen auf: Das Profil eines Licence-Absolventen besteht in einem Basiswissen der deutschen Sprache, Literatur, Kultur und Geschichte, welches erlaubt, sich erstens im Tätigkeitsfeld der Forschung und zweitens im Tätigkeitsfeld des Schuldienstes zu spezialisieren. Geplant war auch ein Studiengang in Übersetzungswissenschaft, der leider in Ermangelung qualifizierter Dozenten nicht umgesetzt werden kann. Trotz der sehr allgemein gehaltenen Formulierung erkennt man zwei der vorgestellten Ansätze für die Germanistik in Afrika wieder: den Ansatz der Germanistik als forschungstheoretische Wissenschaft und die Germanistik als berufsbezogene Fremdsprachenausbildung.

\section{Umsetzung der Zielsetzung der Deutschabteilung}

Die Deutschabteilung möchte also zum einen Nachwuchswissenschaftler und zum anderen Deutschlehrer ausbilden. Nun stellt sich die Frage, ob die Abteilung diese beiden gesetzten Ziele erreicht. Dafür wurde eine Verbleibstudie bei Germanistikabsolventen der Abteilung durchgeführt. Folgende Methode wurde angewandt: Als zu untersuchende Gruppe wurden die Absolventen ausgewählt, die ihr Studium im Hochschuljahr 2003/04 aufgenommen und im Hochschuljahr 2006/07 (folgend als Jahrgang 2003-2007 bezeichnet) abgeschlossen haben. Die in Lomé lebenden Absolventen wurden im Februar 2009 zu einer Diskussionsrunde über ihre derzeitige Lage und ihre Perspektiven eingeladen. Am Ende der Veranstaltung füllten sie einen Fragebogen zu ihrem Verbleib und zur Evaluierung ihres Studiums aus. Außerdem erhielten Absolventen im Inland Togos und die im Ausland lebenden Absolventen über einen Mittler und per E-Mail ebenfalls Fragebögen.

\subsection{Umsetzung des forschungstheoretischen Ansatzes}

Wenden wir uns zunächst dem forschungstheoretischen Ansatz und der Ausbildung von Nachwuchswissenschaftlern zu. Aufgrund eines hochqualifizierten Lehrkörpers besteht an der Deutschabteilung die Möglichkeit, mit einer Promotion abzuschließen. Das Beispiel des Jahrgangs 2003-2007 demonstriert die Entwicklung der Studierendenzahlen bis zur Promotion (siehe Graphik 1): Von 208 Studierenden, die 2003 ihr Deutschstudium aufgenommen hatten, haben 82 nach drei Jahren den ersten berufsqualifizierenden Abschluss 
- die Licence - abgelegt und 63 das C2. C2 bedeutet das 4. Studienjahr, welches eine Voraussetzung für den Maîtrise-Abschluss ist. Um den Maîtrise-Abschluss zu erhalten, müssen die Studierenden eine zweite Voraussetzung erfüllen: das Schreiben und Verteidigen einer Maîtrise-Arbeit. Dies gelingt den wenigsten. Aufgrund der desolaten infrastrukturellen Ausstattung der Bibliotheken fehlt die Forschungsgrundlage. Für die benötigte Literatur sind sie auf einen Forschungsaufenthalt in Deutschland angewiesen. Mit Hilfe von DAADStipendien erhalten jedes Jahr 3-4 Studierende diese Möglichkeit. Diejenigen, die vor Ort versuchen, ihre Maîtrise-Arbeit zu schreiben, brauchen oft mehrere Jahre für ihre Fertigstellung. Bis Februar 2009 hatten von dem die Studie betreffenden Jahrgang nur drei ihre Maitrise-Arbeit verteidigt. Zwei davon setzen ihre akademische Karriere mit dem DEA (Diplôme d'Études Approfondies) fort und planen ihre Promotion. DEA ist das 5. Jahr und gilt als Vorpromotionsstudium, in dem eine Vorarbeit für die Dissertation geleistet wird.

\section{Grafik 1.}

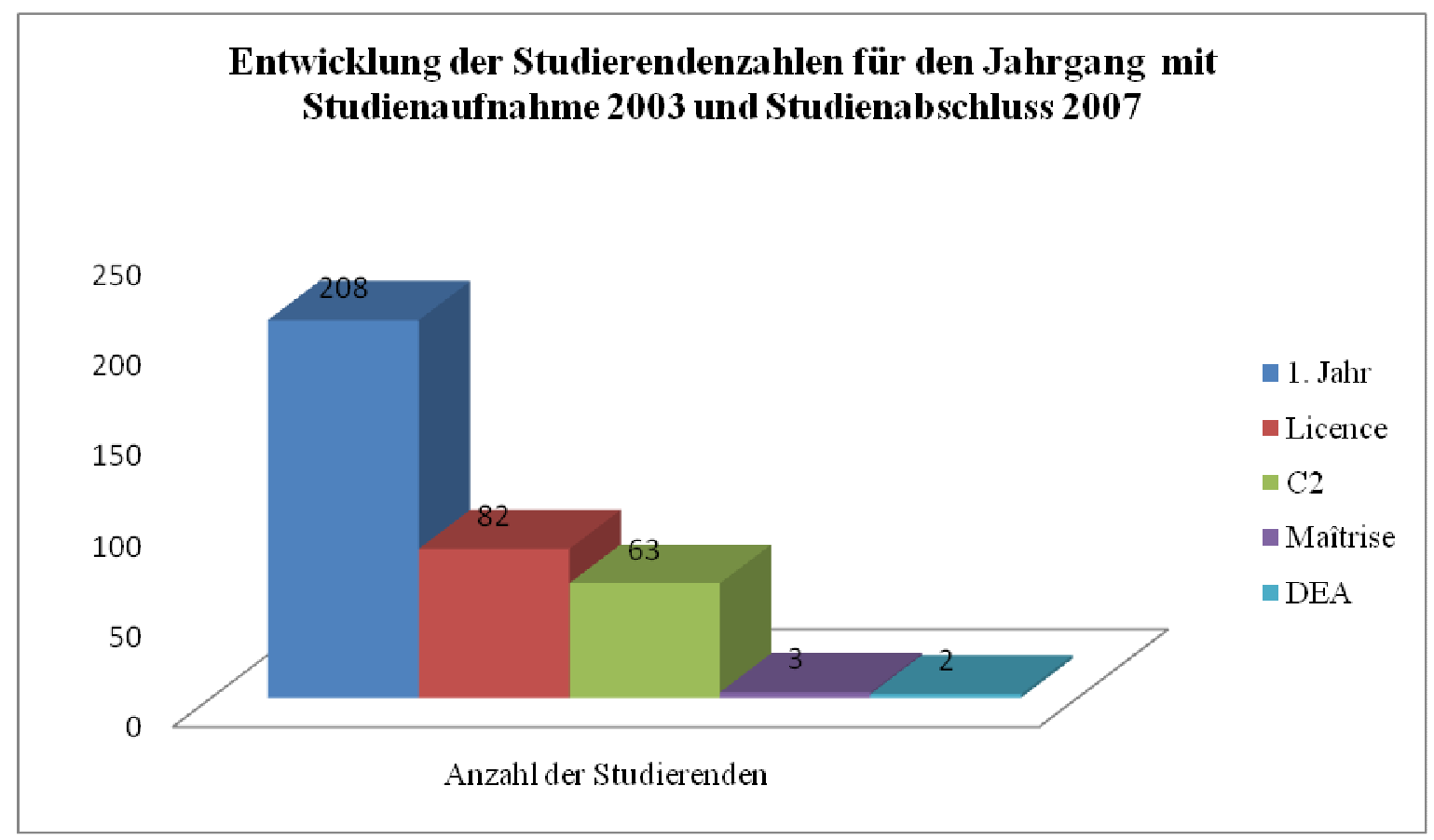

Der wissenschaftliche Nachwuchs wird in forschungswissenschaftliche Tätigkeiten eingebunden. In Forschungsinstitutionen wie Labo-Gerdan von Prof. Glitho, ARTELI von Prof. Oloukpona oder CERGETO von Prof. Ahadji können Studierende ihre Arbeit vorstellen und erhalten dadurch eine wissenschaftliche Orientierung.

Die Abteilung ist folglich in der Lage, Nachwuchswissenschaftler auszubilden. Ihre Einstellungschancen wiederum sind zurzeit begrenzt. Der Bedarf an Dozenten in der Deutschabteilung ist prinzipiell gedeckt, bzw. sind in Ermangelung an Vorlesungssälen keine weiteren Kurse mit neuen Dozenten möglich. Ein erneuter Bedarf würde erst mit der Eröffnung der schon lange geplanten zweiten Deutschabteilung an der Universität Kara im Inland Togos entstehen. 


\subsection{Umsetzung des berufsorientierten Ansatzes}

Der zweite umgesetzte Ansatz ist die berufsbezogene Ausbildung, die sich im Fall von Togo derzeit noch ausschließlich auf die Lehrerausbildung konzentriert. Vorangestellt sei, dass in anderen frankophonen Ländern wie in der Côte d'Ivoire, im Senegal, in Burkina Faso, Mali und Benin Studierende im Anschluss an ihr Germanistikstudium eine pädagogische Hochschule, die ENS (Ecole Nationale Supérieure), besuchen können. In Togo gibt es zwar auch eine pädagogische Hochschule, diese bildet aber nur Realschullehrer aus. Deutsch als Wahlpflichtfach wird jedoch erst am Gymnasium unterrichtet. Somit erhalten Deutschlehrer für das Gymnasium in Togo keine dezidierte Deutschlehrerausbildung, wie es in den anderen frankophonen Ländern üblich ist. Eine Kompensierung erfolgt durch eine pädagogisch/didaktische Ausbildung, die den Deutschstudierenden im 3. und 4. Studienjahr von dem Institut für Erziehungswissenschaften angeboten wird und zwei Seminare von je zwei Semesterwochenstunden umfasst. Nur ein Drittel bis maximal die Hälfte aller Studierenden eines Jahrgangs besuchen diese fakultativ angebotenen Seminare. Das Seminar über beide Semester im 3. Jahr widmet sich der Pädagogik. Im 4. Jahr wird ein Semester lang eine Didaktikvorlesung gehalten, dem ein 4-monatiges Praktikum folgt, in dem die Studierenden in Fünfer-Gruppen an ausgewählten Gymnasien in Lomé hospitieren und auch selbst erste Unterrichtsversuche durchführen. Im Zuge der Professionalisierung im Rahmen eines Reformprozesses soll diese pädagogische Ausbildung effizient umorganisiert werden.

Um ein umfassenderes Bild zum Verbleib der so ausgebildeten Lehrer zu erhalten, wurde die Umfrage für die folgenden zwei Aspekte (die Erfüllung der beruflichen Erwartungen und die Berufswünsche zu Beginn des Studiums) auf Lehrer älter als der Jahrgang 2003-2007 in Lomé und im Inland ausgedehnt. Von den insgesamt 46 befragten Deutschlehrern haben nur etwas mehr als die Hälfte (25) die eben beschriebene pädagogische Ausbildung genossen.

Auf die Frage, ob ihre derzeitige Tätigkeit die beruflichen Erwartungen und Vorstellungen erfüllt, die sie während des Studiums hatten, antworteten 57 Prozent (24) „,gar nicht bzw. ein wenig“, ein knappes Drittel von 31 Prozent (13) gab „mittel“ an und nur 12 Prozent (5) der Lehrer ,in hohem Maße“. 


\section{Grafik 2.}

\section{Erfüllung der beruflichen Erwartungen}

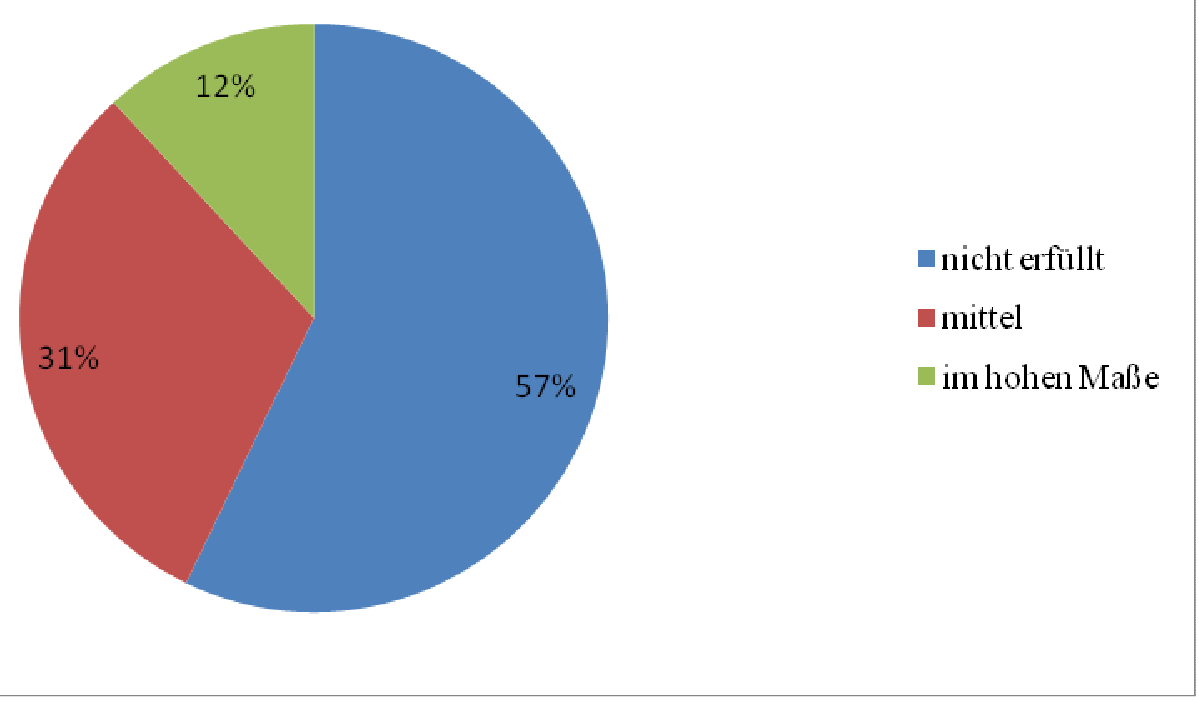

Die große Unzufriedenheit erklären sie zum einen damit, dass sie eigentlich andere Berufsziele anstrebten. Schaut man sich die Berufsziele der befragten Lehrer zu Beginn des Studiums an (siehe Grafik 3), ergibt sich folgendes Bild: Von den befragten Lehrern wollten 44 Prozent (18) Dolmetscher werden, je 10 Prozent (4) Journalist bzw. Dozent, 7 Prozent (3) Diplomat, 3 Prozent (1) Schriftsteller, 2 Prozent (1) nach Deutschland fliegen. Nur ein Viertel, genauer 24 Prozent (10), wollte wirklich Lehrer werden.

\section{Grafik 3.}

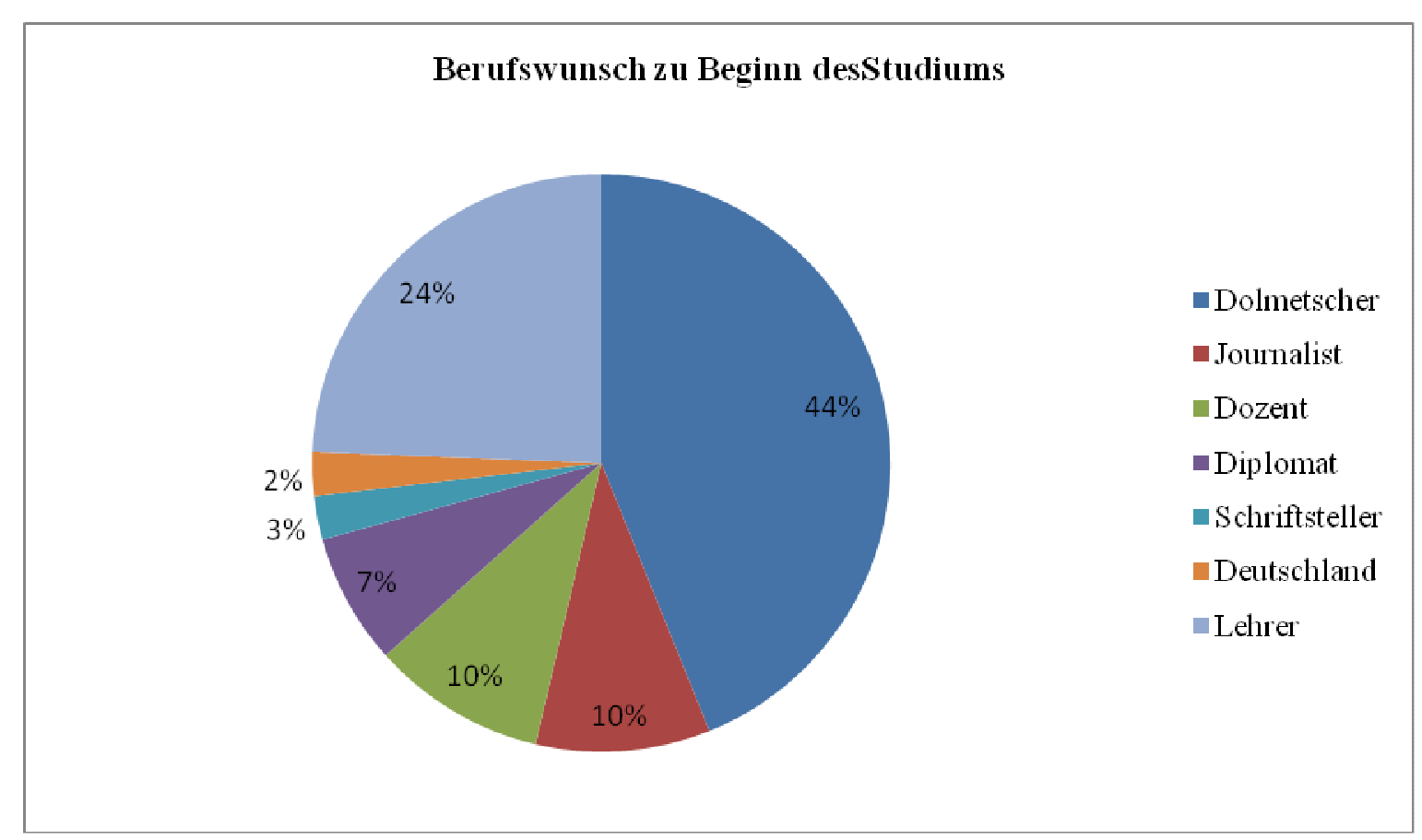


15 Prozent (14) geben an, ihr Berufsziel noch nicht erreicht zu haben. Sie würden gern ihr Studium fortsetzen und - die forschungstheoretische Richtung einschlagen. Acht Deutschlehrer bemängeln infrastrukturelle und finanzielle Unzulänglichkeiten in ihrem Beruf. Drei Lehrer sind nicht zufrieden, da es nur ein Job sei, der keine Freude macht, bzw. anstrengender ist als gedacht. Zwei Lehrer äußerten, der Deutschunterricht habe nichts mit der afrikanischen Realität zu tun. Nur bei drei Deutschlehrern entspricht der Beruf den Vorstellungen: Sie hatten vor, Deutschlehrer zu werden und sind es wirklich geworden.

Die häufigsten Probleme beim Berufsstart sind interessanterweise 12 Nennungen des „Gefühls der Unterforderung“. Eigentlich wäre hier die Antwort zu erwarten, im Studium nicht gut auf den späteren Beruf vorbereitet worden zu sein. Analysiert man die Antwort jedoch genauer, kommt man zu dem Schluss, dass Studierende eine höher gestellte bzw. akademische Karriere anvisiert haben. Das Curriculum der Deutschabteilung setzt neben der sprachpraktischen Ausbildung einen Schwerpunkt auf die Literaturwissenschaft. Ein Großteil der Kurse widmet sich der vergleichenden, klassischen und modernen Literatur und der Gegenwartsliteratur. Dadurch werden Studierende forschungstheoretisch orientiert und können sich dann im Gymnasium im Anfängerunterricht für Deutsch unterfordert fühlen.

Um die Frage im Titel meines Beitrags zu beantworten, möchte ich zum Schluss den Verbleib des untersuchten Jahrgangs 2003-07 (siehe Grafik 5) analysieren: Von den befragten Absolventen arbeiten bis jetzt 22 Prozent (16) als Lehrer. Davon sind genau die Hälfte in staatlichen und die andere Hälfte in privaten Gymnasien beschäftigt. Hinzufügen muss man, dass die Stundenzahl an den privaten Gymnasien oft verschwindend gering ist. Lehrer arbeiten zwischen 3 bis 16 Stunden die Woche. 13 Prozent (9) der Absolventen sind in einem anderweitig festen Beschäftigungsverhältnis. Davon sind zwei am Goethe-Institut in der Bibliothek und als projektbezogener Assistent für die Expertin für Unterricht tätig, eine in der deutschen Botschaft und einer als Übersetzer in der Regierung. Ein Au-Pair-Vermittler, ein Steuerbeamter, ein Lagerverwalter in einer Düngerfirma und ein Aufseher in einer Sicherheitsagentur vervollständigen das Spektrum. Über ein Drittel der Absolventen gehen also einem deutsch-relevanten Beruf nach, bzw. befinden sich in einer festen Anstellung mit einem für togoische Verhältnisse angemessenen Gehalt. 15 Prozent (11) gehen Gelegenheitsjobs nach. Diese Gelegenheitsjobs umfassen neben vier Nachhilfe-Lehrern für Deutsch, zwei Händlerinnen, einen Fotografen, einen Haarsträhnen-Verkäufer, zwei MotoTaxi-Fahrer, einen Radiomoderator, einen Tänzer und einen Angestellten in der Verwaltung an der Universität. 19 Prozent (14) haben ein Zweitstudium begonnen (vier davon in Deutschland). Die Fächerwahl für das Zweitstudium reicht von Erziehungswissenschaften (5), Englisch (2), Soziologie (3), Jura (1), Interkulturelle Kommunikationswissenschaften (1), Übersetzen/Dolmetschen (1) bis zu Politikwissenschaften (1). Die vier letztgenannten sind die in Deutschland gewählten Studiengänge, von denen die letzen drei nicht in dieser Form an der einheimischen Universität studiert werden können. Auffällig ist auch, dass ein Drittel ein Zweitstudium in Erziehungswissenschaften aufnimmt. Diese Wahl deutet auf einen Fortbildungsbedarf in diesem Bereich hin, der während des Erststudiums noch nicht genügend gedeckt wurde. 6 Prozent (4) wählten eine Fortbildung bzw. ein Praktikum in einem Sekretariat, bei der Sozialversicherungsanstalt und am Flughafen. 25 Prozent (18) sind arbeitslos, obwohl acht von ihnen die angebotene Lehrerausbildung in den Erziehungswissenschaften wahrgenommen haben. 


\section{Grafik 4.}

\section{Verbleib der Germanistikabsolventen des Jahrgangs mit Studienaufnahme 2003 und Studienabschluss 2007}

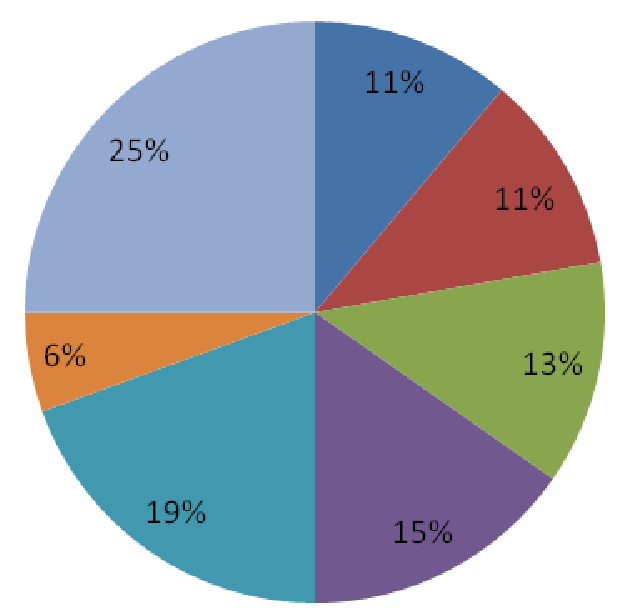

Deutschlehrer staatliches Gymnasium

Deutschlehrer privates Gymnasium

- festes Beschäftigungsverhältnis

- Gelegenheitsjob

- Zweitstudium

Fortbildung und Praktika

arbeitslos

Dieses Ergebnis präsentiert trotz der 25 Prozent Arbeitslosigkeit ein relativ hoffnungsvolles Bild. In Togo existiert generell eine verhältnismäßig hohe Arbeitslosenquote (eine genaue Zahl liegt in Ermangelung einer offiziellen statistischen Erfassung nicht vor). Die Absolventen beweisen trotz der schwierigen Arbeitsmarktlage Kreativität und Flexibilität auf der Suche nach Beschäftigung. Zu beachten ist ebenfalls, dass sich die untersuchte Gruppe erst ein knappes Jahr auf dem Arbeitsmarkt befindet. Die Einstellung von Deutschlehrern für die staatlichen Gymnasien wird zentral von der Regierung durch alle 2-4 Jahre stattfindende Concours de recrutement d'enseignants organisiert. Beim letzten Auswahlverfahren 2008 sind 50 Germanistikabsolventen von der Regierung in den Schuldienst aufgenommen worden, u.a. auch die 8 Deutschlehrer der staatlichen Gymnasien von der untersuchten Gruppe. Positiv zu bewerten ist auch, dass die Hälfte der Befragten Deutsch noch einmal studieren würde.

\section{Perspektiven}

Die Deutschabteilung hat, wie andere afrikanische frankophone Länder auch, eine einmalige Chance der Reformierung. Genauso wie der Bologna-Prozess in Europa, so vollzieht sich auch in den frankophonen Ländern ein Bologna-Prozess à l'africaine - die LMD-Reform. Die Reform umfasst sowohl formale als auch inhaltliche Veränderungen in der Studienstruktur. Formale Veränderungen sind die Einführung der Semestrialisierung, der Modularisierung und der Kapitalisierung von Kreditpunkten für die neu geschaffenen drei aufeinander aufbauenden Zyklen, bzw. akademischen Abschlüssen in der Hochschulbildung. LMD steht für diese 3 Zyklen: Licence, Master, Doctorate. Als inhaltliche Veränderungen spielen für diesen Beitrag die verstärkte Professionalisierung und Transdisziplinarität eine übergeordnete Rolle, da diese dem dritten Ansatz des berufsbezogenen und fachsprachlichen Fremdsprachenangebots eine wesentlich stärkere Gewichtung einräumen und den Absolventen größere Einstellungschancen in Aussicht stellen. 
Professionalisierung bedeutet das Einbringen von Komponenten der Allgemeinbildung, aber auch Anteile der Spezialisierung und des Praxisbezugs in das Curriculum der Deutschabteilung. Neben speziellen Fachkenntnissen und methodischen Fähigkeiten sind vermehrt Schlüsselqualifikationen, etwa soziale Kompetenzen, zu vermitteln. Dazu gehören allgemein-wissenschaftliche Fähigkeiten, wie weitere Fremdsprachenkenntnisse, der zielgerichtete Umgang mit wissenschaftlicher Software oder rhetorisches Geschick, aber auch überfachliche Kompetenzen, etwa die Fähigkeit zur Teamarbeit, das Planen und Organisieren von Projekten oder die Umsetzung theoretischer Kenntnisse in die Praxis. Diese Fähigkeiten werden an der Deutschabteilung zunehmend in Kursen wie Technique d'Expression Orale und in gruppenbasierten Hausaufgaben erworben. Die Studierenden erhalten schon jetzt vermehrt projektbezogene Aufgaben. Es wird weiterhin zumindest theoretisch angedacht, Informatik in das Transversale-Modul bis ins 4. Jahr zu integrieren. Der Erwerb von Schlüsselqualifikationen dient der Professionalisierung des Studiengangs, der zur Erleichterung des Berufseinstiegs der Germanistik-Absolventen führen kann.

Mit der LMD-Reform hat die Deutschabteilung auch gute Möglichkeiten für den Ausbau einer Transdisziplinarität. Zum einen ließe sich die Deutschlehrerausbildung in Zusammenarbeit mit den Erziehungswissenschaften ausbauen, aber auch die Vernetzung mit Wirtschaft, Soziologie, Medizin und Jura bieten Germanistikabsolventen Perspektiven für einen Berufseinstieg außerhalb des Lehrerberufs. Das Ziel der Transdisziplinarität ist die Diversifizierung der Absolventen-Profile. Bis jetzt belegen alle Studierenden genau die gleichen Lehrveranstaltungen in den beiden Spezialisierungsmöglichkeiten innerhalb der Germanistik. Die zwei Spezialisierungsmöglichkeiten in Littérature und Civilisation dienen jedoch nur einer der beiden Zielsetzungen der Deutschabteilung - der Umsetzung des forschungstheoretischen Ansatzes. Im Zuge der Reform plant die Abteilung, einen forschungsorientierten und einen berufsorientierten Master einzuführen. Die Einrichtung eines berufsorientierten Masters (außer Deutschlehrer am Gymnasium) in Verbindung mit anderen Fakultäten würde auch der Aufhebung der Stigmatisierung der Germanistikabsolventen dienen: In den Augen potentieller Arbeitgeber und des Arbeitsministeriums können Germanisten nur Lehrer werden. Hier könnte man sich an dem LATA-Studiengang im Senegal oder an den Studiengängen des GIL orientieren. Diesbezüglich wurde in der Abteilung eine Kommission gegründet, die mögliche berufsorientierte Master entwickeln soll. In Erwägung wird ein berufsorientierter Master in Übersetzen/Dolmetschen gezogen. Der Mangel an qualifizierten Lehrkräften soll vorerst mit Gastdozenturen kompensiert werden. Damit könnte man beruflichen Erwartungen der Studierenden (siehe Studie) gerecht werden und gleichzeitig den von deutschen Institutionen in Lomé angemeldeten Bedarf decken.

Zusammenfassend lässt sich feststellen, dass zwei der drei entwickelten Ansätze zur Etablierung des Deutschen in Afrika an der Deutschabteilung der Universität Lomé Anwendung finden. Die Umsetzung des forschungstheoretischen Ansatzes erfolgt in der Abteilung aufgrund des hochqualifizierten Lehrkörpers unter besten Voraussetzungen. Es stellt sich aber die Frage nach dem Bedarf für Nachwuchswissenschaftler. Viele von ihnen werden in den Schuldienst übernommen. Die Umsetzung des berufsorientierten Ansatzes ist derzeit noch auf das Berufsfeld des Deutschlehrers am Gymnasium konzentriert. Die Ausbildung dafür hat noch Potential zu Verbesserungen. Perspektiven bietet die bis 2011 umzusetzende LMD-Reform, die durch eine verstärkte Professionalisierung und 
Transdisziplinarität eine Erweiterung der Tätigkeitsfelder und eine fundiertere Deutschlehrerausbildung in Aussicht stellt.

\section{Bibliographie}

Bedi, L.E. 2006. Deutsch in Afrika: Vergangenheit, Gegenwart, Zukunft. Hamburg: Verlag Dr. Kovač.

Diop, E.H.I. 2000. Das Selbstverständnis von Germanistik und Deutschunterricht im frankophonen Afrika. Vom kolonialen Unterrichtsfach zu eigenständigen Deutschlandstudien und zum praxisbezogenen Lernen. Frankfurt a.M.: Peter Lang Verlag.

Ihekweazu, E. 1984. Länderbericht Nigeria. Jahrbuch Deutsch als Fremdsprache 10: 264274.

Ihekweazu, E. 1987. German Studies in Nigeria and the Inter-Cultural Concept. Études germano-africaines. Revue annuelle du Département de Langues et Civilisations Germaniques de la Faculté des Lettres et Sciences Humaines de l'Université de Dakar 5: 65-72.

Kreutzer, L. 1989. Literatur und Entwicklung. Studien zu einer Literatur der Ungleichzeitigkeit. Frankfurt a.M.: Fischer Verlag.

Kreutzer, L. 1989. Legitimationsprobleme einer Germanistik in Afrika. In P. Kasprzyk und N. Ndong (Hgg.) Afrikanische Germanistik. Eine Dokumentation. Bonn: DAAD. pp. 104109.

Kreutzer, L. 1998. Germanistik als Entwicklungswissenschaft. Zeitschrift für Germanistik. Neue Folge 8(3): 529-537.

Kreutzer, L. 2009. Goethe in Afrika. Die interkulturelle Literaturwissenschaft der „École de Hanovre " in der afrikanischen Germanistik. Hannover: Wehrhahn Verlag.

Ndong, N. 1993. Entwicklung, Interkulturalität und Literatur. Überlegungen zu einer afrikanischen Germanistik als interkulturelle Literaturwissenschaft. München: Iudicium Verlag.

Sadji, A.B. 1983. Prolégomènes à propos de l'enseignement de l'allemand et des études germanistiques au Sénegal. Études germano-africaines. Revue annuelle du Département de Langues et Civilisations Germaniques de la Faculté des Lettres et Sciences Humaines de l'Université de Dakar 1: 17-27.

Sadji, A.B. 1984. Deutschunterricht und Germanistik in Senegal. Jahrbuch Deutsch als Fremdsprache 10: 75-85.

Simo, D. 1984. Deutschstudium in Kamerun. Jahrbuch Deutsch als Fremdsprache 10: 275280.

Simo, D. 1999. Interkulturelle Germanistik und Postkolonialität in Afrika. Jahrbuch Deutsch als Fremdsprache 25: 349-363.

Sow, A. 1986. Germanistik als Entwicklungswissenschaft. Überlegungen zu einer Literaturwissenschaft des Faches „Deutsch als Fremdsprache“ in Afrika. Hildesheim: Georg Olms Verlag.

Wierlacher, A. (Hg.) 1985. Das Fremde und das Eigene. Prolegomena zu einer interkulturellen Germanistik. München: Iudicium Verlag.

\section{Biographical note}

Eva Hamann studied Ethnology and American Studies at Leipzig University and at the University of Miami, as well as German as a Foreign Language at Leipzig University. From 2004 to 2009 she was a DAAD-lecturer at the Université de Lomé in Togo. Since 2009 she has been at the Herder Institute at Leipzig University. 